\title{
Painlevé integrability and multisoliton solutions of a generalized KdV system
}

\author{
Pinki Kumari ${ }^{1, *}$, R.K. Gupta ${ }^{1,2, * *}$, and Sachin Kumar $^{1, * * *}$ \\ ${ }^{1}$ Department of Mathematics and Statistics, Central University of Punjab, Bathinda, Punjab, India \\ ${ }^{2}$ Department of Mathematics, Central University of Haryana, Mahendergarh, Haryana, India
}

\begin{abstract}
The integrability of a generalized KdV model, which has abundant physical applications in many fields, is investigated by employing Painlevé test. Eventually, we discover a new generalized P-type KdV model in sense of WTCKruskal method. Subsequently, Hereman's simplified bilinear method is used to examine the integrability of the resulted model. As a result, multiple soliton solutions of newly discovered model are formally obtained.
\end{abstract}

\section{Introduction}

A series of single component as well as coupled $\mathrm{KdV}$ equations and their generalizations has been studied widely [1-4, 6, 22]. For more than a century, the study of $\mathrm{KdV}$ equation is gaining popularity because of its broad applications in fluid dynamics. Also numerous theoretical models in other fields of physics have been discussed through KdV equation. For history, features and applications of KdV equation, one can refer [7].

Nowadays it is seen that the investigation of generalized system of equations with arbitrary coefficients rather than the specified ones has become hot topic as they reveal some new features, properties about the model for several different parameters which are more helpful in scientific as well as theoretical study $[8,9]$.

In this study, we propose a generalized coupled KdV system

$$
\begin{gathered}
u_{t}+a u_{x x x}+b u u_{x}+c v u_{x}=0, \\
v_{t}+p v_{x x x}+q v v_{x}+r u v_{x}=0,
\end{gathered}
$$

to examine its integrability via Painlevé test and simplified form of Hirota's bilinear method. Here coefficients $a, b, c, p, q$ and $r$ are nonzero random constants.

Integrable partial differential equations (PDEs), mainly ones having soliton solutions, have had a very consequential impact on phenomenology and theory [10]. Painlevé integrability is an important feature of a complete integrable PDE [11] i.e. a P-type PDE is likely to be integrable. As Ablowitz et al. $[12,13]$ stated that all similarity reductions of a integrable PDE have Painlevé property if their general solutions do not have movable singularities except on poles in the complex plane. In a nutshell, Painlevé test is used to inspect the singularity structure of solutions of differential equations.

\footnotetext{
*e-mail: yadav.pk1403@gmail.com

**e-mail: rajeshateli@gmail.com

***e-mail: sachin1jan@yahoo.com
} 
No doubt, integrable systems have performed a significant role in physical sciences and proved as a fundamental topic in soliton theory to explore more integrable models. To find new integrable models, study of extensions of existing integrable systems is an effective way. Complete integrability of a nonlinear evolution equation can be examined by many techniques like the Painlevé analysis, the Lax pair, the Bäcklund transformation method, the Hirota bilinear method etc. Among existing techniques, Hereman' simplified bilinear method [14], which requires determination of multi soliton solutions, is more reliable, practical and more powerful in handling the integrable nonlinear evolution systems. For study on multi soliton solutions, one can refer [15-18].

\section{Painlevé Test}

In this section, we test the Painlevé integrability of system (1) in sense of WTC algorithm. Weiss et al. suggested WTC (Weidss Tabor Carnevale) method [19] of Painlevé analysis of PDEs. The method is algorithmic, effective and does not require reduction to ODEs for testing PDEs. Also by-product of this test can be used to construct Backlund transformation and exact solutions of PDEs. The effectiveness of the method can be seen in refs. [1, 19-21].

Following [19], we assume that system (1) has following Laurent expansion

$$
\begin{aligned}
& u(x, t)=\phi^{\alpha_{1}}(x, t) \sum_{i=0}^{\infty} u_{i}(x, t) \phi^{i}(x, t), \\
& v(x, t)=\phi^{\alpha_{2}}(x, t) \sum_{i=0}^{\infty} v_{i}(x, t) \phi^{i}(x, t),
\end{aligned}
$$

where $u_{i}(x, t), v_{i}(x, t)$ are analytic functions in the neighbourhood of movable singular manifold $\phi(x, t)$ and

$u_{0}(x, t) \neq 0, v_{0}(x, t) \neq 0$. For leading order analysis, we substitute $u \approx u_{0} \phi^{\alpha_{1}}, v \approx v_{0} \phi^{\alpha_{2}}$ in system (1). Then equating the most dominant terms, we get

$$
\begin{aligned}
& \alpha_{1}=-2, \alpha_{2}=-2, \\
& u_{0}(x, t)=-\frac{12 \phi_{x}^{2}(a q-c p)}{(b q-c r)}, \\
& v_{0}(x, t)=\frac{12 \phi_{x}^{2}(a r-b p)}{(b q-c r)} .
\end{aligned}
$$

Next task is to find resonance points for $\alpha_{1}=-2$,

$\alpha_{2}=-2, u_{0}, v_{0}$. So by plugging (2) into (1) along with $\alpha_{1}=-2, \alpha_{2}=-2, u_{0}, v_{0}$ and then comparing the coefficients of term $\phi^{i-5}$, we get the following polynomial in $i$ :

$$
\begin{aligned}
& (i+1)(i-4)(i-6)\left(i-\left(\frac{s}{3 a p(b q-c r)}+\frac{13 a p(b q-c r)}{s}+3\right)\right)\left(i-\left(\frac{-s}{6 a p(b q-c r)}\right.\right. \\
& \left.\left.-\frac{13 a p(b q-c r)}{2 s}+3+\iota \frac{\sqrt{3}}{2}\left(\frac{s}{3 a p(b q-c r)}-\frac{13 a p(b q-c r)}{s}\right)\right)\right)\left(i-\left(\frac{-s}{6 a p(b q-c r)}\right.\right. \\
& \left.\left.-\frac{13 a p(b q-c r)}{2 s}+3-\iota \frac{\sqrt{3}}{2}\left(\frac{s}{3 a p(b q-c r)}-\frac{13 a p(b q-c r)}{s}\right)\right)\right) \lambda=0,
\end{aligned}
$$


where

$$
\begin{aligned}
s= & \left(324 a^{2} q r-162 a b p q-486 a c p r+324 b c p^{2}\right. \\
& +3 \sqrt{3}\left\{3888 a^{4} q^{2} r^{2}-3888 a^{3} b p q^{2} r-11664 a^{3} c p q r^{2}\right. \\
& -1225 a^{2} b^{2} p^{2} q^{2}+18002 a^{2} b c p^{2} q r+6557 a^{2} c^{2} p^{2} r^{2} \\
& \left.\left.\left.-3888 a b^{2} c p^{3} q-11664 a b c^{2} p^{r}+3888 b^{2} c^{2} p^{4}\right\}^{\frac{1}{2}}\right) a^{2} p^{2}(b q-c r)^{2}\right)^{\frac{1}{3}} .
\end{aligned}
$$

So, the resonance points for the only branch are

$$
\begin{aligned}
& -1,4,6, \frac{s}{3 a p(b q-c r)}+\frac{13 a p(b q-c r)}{s}+3, \frac{-s}{6 a p(b q-c r)} \\
& -\frac{13 a p(b q-c r)}{2 s}+3+\iota \frac{\sqrt{3}}{2}\left(\frac{s}{3 a p(b q-c r)}-\frac{13 a p(b q-c r)}{s}\right) \\
& \frac{-s}{6 a p(b q-c r)}-\frac{13 a p(b q-c r)}{2 s}+3-\iota \frac{\sqrt{3}}{2}\left(\frac{s}{3 a p(b q-c r)}-\frac{13 a p(b q-c r)}{s}\right) .
\end{aligned}
$$

We can clearly see that the branch contains non-integer resonances. So Painlevé test for arbitrary choice of coefficients of system (1) does not pass. But, with the help of (5) and (6), we find a condition $p=a, q=c, r=b$ that yields the integer type resonances as $-1,0,2,4$, 6,7 . So we obtain the following system that is likely to be P-integrable:

$$
\begin{aligned}
u_{t}+a u_{x x x}+b u u_{x}+c v u_{x} & =0, \\
v_{t}+a v_{x x x}+c v v_{x}+b u v_{x} & =0 .
\end{aligned}
$$

Now to test the Painlevé integrability of (7), we need to verify the compatibility conditions. By substituting (2), truncated up to largest resonance i.e. $i=7$, into system (1) and collecting the coefficients of polynomial in $\phi$, we get the following resonance conditions:

$$
\begin{aligned}
& u_{0}(t)=-\frac{12 a+c v_{0}(t)}{b}, \quad v_{0}(x, t)=v_{0}(t), \\
& u_{1}(t)=0, \quad v_{1}(t)=0, \\
& u_{2}(t)=-\frac{c v_{2}(t)}{b}+\frac{\psi^{\prime}(t)}{b}, \quad v_{2}(t)=v_{2}(t), \\
& u_{3}(t)=-\frac{c v_{0_{t}}}{12 b a}, \quad v_{3}(t)=\frac{v_{0_{t}}}{12 a}, \\
& u_{4}(t)=-\frac{\left(12 a+c v_{0}(t)\right) v_{4}(t)}{b v_{0}(t)}+\frac{\psi^{\prime}(t)}{b}, v_{4}(t)=v_{4}(t), \\
& u_{5}(t)=-\frac{c v_{0}(t) \psi_{t t}(t)-3 a c v_{2}+15 a \psi_{t t}(t)}{90 b a^{2}}, v_{5}(t)=-\frac{v_{0} \psi_{t t}-3 a v_{2 t}}{90 a^{2}}, \\
& u_{6}(t)=-\frac{\left(12 a+c v_{0}(t)\right) v_{6}(t)}{b v_{0}(t)}+\frac{v_{0_{t t}}(t)}{b a v_{0}(t)}, \quad v_{6}(t)=v_{6}(t), \\
& u_{7}(t)=\frac{-\left(12 a+c v_{0}(t)\right) v_{6}(t)}{b v_{0}(t)}+\frac{v_{4_{t}}(t) v_{0}(t)-v_{4}(t) v_{0_{t}}(t)}{2 b v_{0}^{2}}, v_{7}(t)=v_{7}(t) .
\end{aligned}
$$

The functions $\psi, v_{0}, v_{2}, v_{4}, v_{6}$ and $v_{7}$ in resonance conditions are arbitrary and depending on $t$. It should be noted that Kruskal guage is applied for singular manifold, i.e,

$$
\phi(x, t)=x-\psi(t), u_{r}(x, t)=u_{r}(t), v_{r}(x, t)=v_{r}(t) .
$$




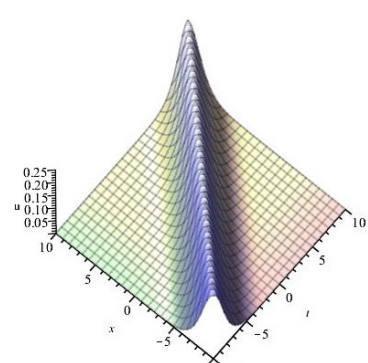

(a)

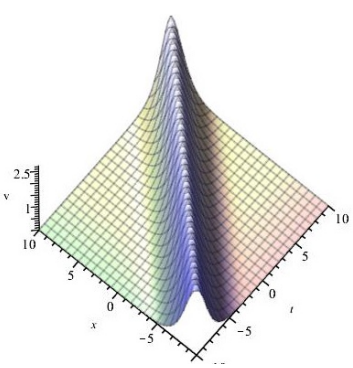

(e)

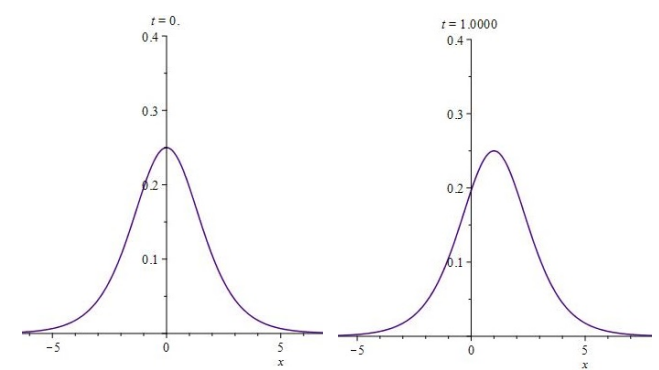

(c)

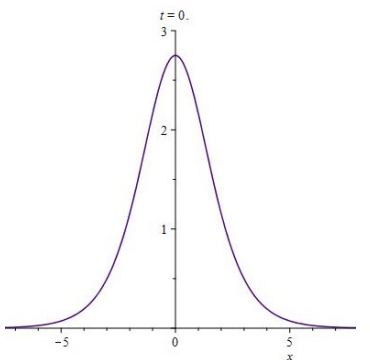

(f)

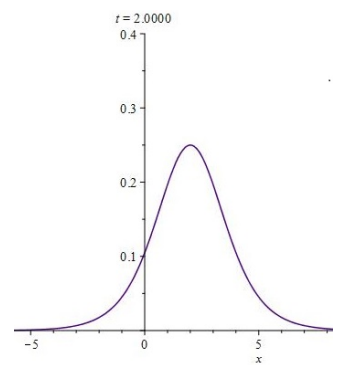

(d)

Figure 1: Profiles of the 1-solitons of the system (7).

As a sufficient number of arbitrary functions exist for the solutions of (7), compatibility conditions are satisfied identically. This proves that system (7) is Painlevé integrable. So, we have determined a new generalized P-integrable KdV system (7). In the next section, we will use the simplified form of Hirota's method to get more information on integrability of system (7).

\section{Multi-soliton Solutions}

In this part, the simplified form of Hirota's method, suggested by Hereman et al. [14], is used to explore multiple soliton solution of (7) and the determination of $\mathrm{N}$-soliton solutions will lead to integrability of the system. The Hereman's simplified method does not depend on Hirota bilinear operator unlike Hirota's method and uses the employment of auxiliary function $\mathrm{f}(\mathrm{x}, \mathrm{t})$.

To obtain the dispersion relation for (7), we insert

$$
u(x, t)=e^{\theta_{i}}, \quad v(x, t)=e^{\theta_{i}}, \theta_{i}=k_{i} x-c_{i} t
$$

into the linear terms of (7). The resulting equation gives

$$
c_{i}=a k_{i}^{3} .
$$

So, the phase variables read as

$$
\theta_{i}=k_{i} x-a k_{i}^{3} t
$$




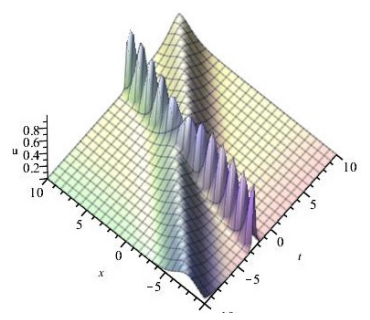

(a)

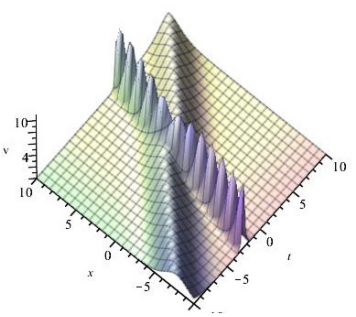

(e)

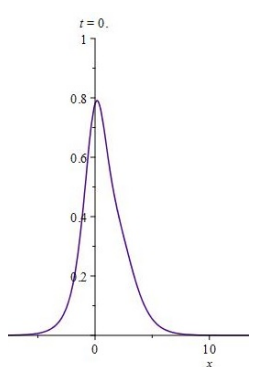

(b)

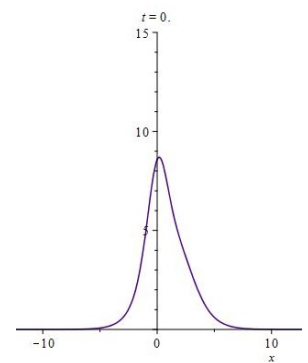

(f)

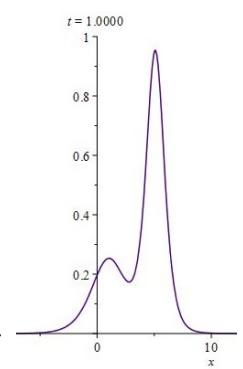

(c)

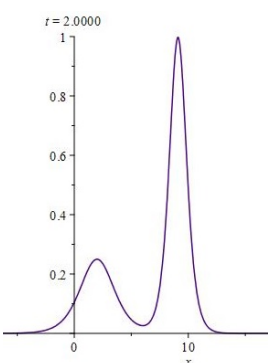

(d)

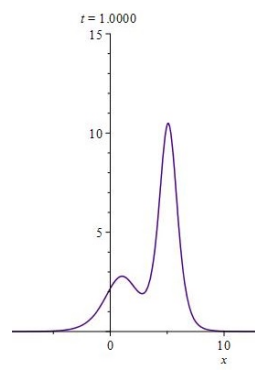

(g)

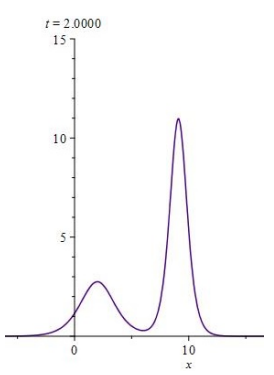

(h)

Figure 2: Profiles of the 2-solitons of the system (7).

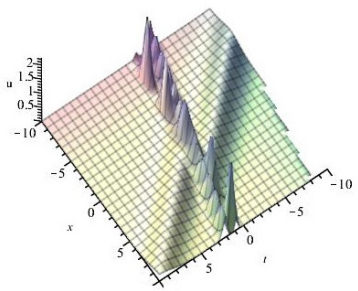

(a)

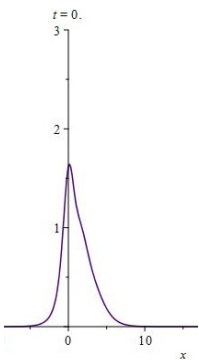

(b)

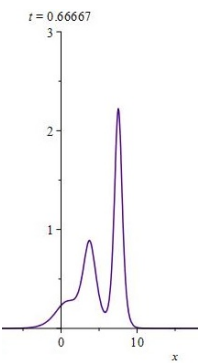

(c)

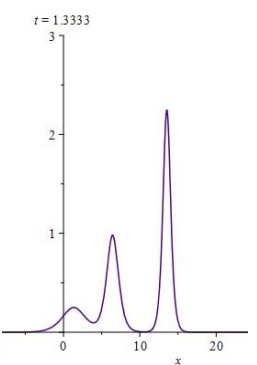

(d)

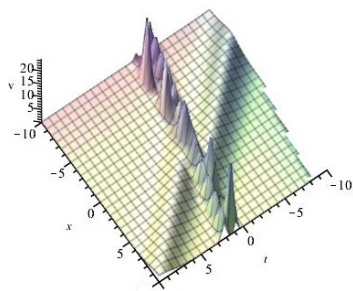

(e)

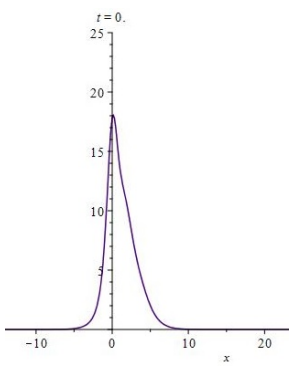

(f)

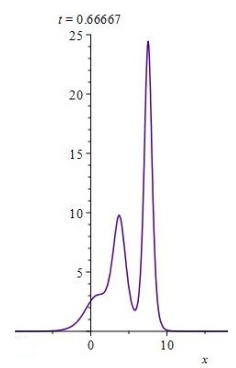

(g)

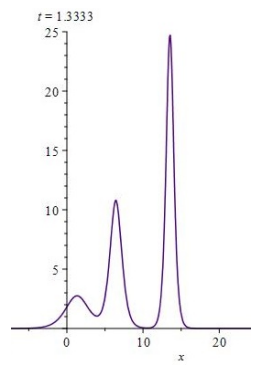

(h)

Figure 3: Profiles of the 3-solitons of the system (7). 
Next, we adopt the following transformations to determine multi soliton solutions of (7):

$$
\begin{aligned}
& u(x, t)=R_{1}(\ln f(x, t))_{x x}=R_{1} \frac{f f_{x x}-f_{x}^{2}}{f^{2}}, \\
& v(x, t)=R_{2}(\ln f(x, t))_{x x}=R_{2} \frac{f f_{x x}-f_{x}^{2}}{f^{2}},
\end{aligned}
$$

where $R_{1}, R_{2}$ are constants to be known later.

For a 1-soliton solution, the auxiliary function $f(x, t)$ is set as

$$
f(x, t)=1+e^{\theta_{1}} .
$$

Substituting (14), (13) into (7), and solving for $R_{1}$ and $R_{2}$, we get

$$
R_{1}=\text { free constants, } R_{2}=\frac{12 a-b R_{1}}{c} .
$$

Consequently, we get the following 1-soliton solutions of (7):

$$
\begin{aligned}
& u(x, t)=\frac{k_{1}^{2} R_{1} e^{k_{1} x-a k_{1}^{3} t}}{\left(1+e^{\left.k_{1} x-a k_{1}^{3} t\right)^{2}}\right.}, \\
& v(x, t)=\frac{12 a-b R_{1}}{c} \frac{k_{1}^{2} e^{k_{1} x-a k_{1}^{3} t}}{\left(1+e^{\left.k_{1} x-a k_{1}^{3} t\right)^{2}}\right.} .
\end{aligned}
$$

For 2-soliton solutions, we set the auxiliary function as

$$
f(x, t)=1+e^{\theta_{1}}+e^{\theta_{2}}+a_{12} e^{\theta_{1}+\theta_{2}},
$$

that, with the help of (13), yields the following solution of (7):

$$
\begin{aligned}
u(x, t)= & \frac{R_{1}}{\left(1+e^{k_{1} x-a k_{1}^{3} t}+e^{k_{2} x-a k_{2}^{3} t}+e^{\left.k_{1} x-a k_{1}^{3} t+k_{2} x-a k_{2}^{3} t\right)^{2}}\right.} \\
& \times\left[k_{1}^{2} e^{k_{1} x-a k_{1}^{3} t}+k_{2}^{2} e^{k_{2} x-a k_{2}^{3} t}+2\left(k_{1}-k_{2}\right)^{2} \times e^{k_{1} x-a k_{1}^{3} t+k_{2} x-a k_{2}^{3} t}+k_{2}^{2} \frac{\left(k_{1}-k_{2}\right)^{2}}{\left(k_{1}+k_{2}\right)^{2}}\right. \\
& \left.\times e^{k_{1} x-a k_{1}^{3} t+k_{2} x-a k_{2}^{3} t}+k_{1}^{2} \frac{\left(k_{1}-k_{2}\right)^{2}}{\left(k_{1}+k_{2}\right)^{2}} \times e^{k_{1} x-a k_{1}^{3} t+2 k_{2} x-2 a k_{2}^{3} t}\right], \\
v(x, t)= & \frac{12 a-b R_{1}}{c\left(1+e^{k_{1} x-a k_{1}^{3} t}+e^{k_{2} x-a k_{2}^{3} t}+e^{\left.k_{1} x-a k_{1}^{3} t+k_{2} x-a k_{2}^{3} t\right)^{2}}\right.} \\
\times & \times\left[k_{1}^{2} e^{k_{1} x-a k_{1}^{3} t}+k_{2}^{2} e^{k_{2} x-a k_{2}^{3} t}+2\left(k_{1}-k_{2}\right)^{2} \times e^{k_{1} x-a k_{1}^{3} t+k_{2} x-a k_{2}^{3} t}+k_{2}^{2} \frac{\left(k_{1}-k_{2}\right)^{2}}{\left(k_{1}+k_{2}\right)^{2}}\right. \\
& \left.\times e^{k_{1} x-a k_{1}^{3} t+k_{2} x-a k_{2}^{3} t}+k_{1}^{2} \frac{\left(k_{1}-k_{2}\right)^{2}}{\left(k_{1}+k_{2}\right)^{2}} \times e^{k_{1} x-a k_{1}^{3} t+2 k_{2} x-2 a k_{2}^{3} t}\right],
\end{aligned}
$$

with the phase shift by

$$
a_{12}=\frac{\left(k_{1}-k_{2}\right)^{2}}{\left(k_{1}+k_{2}\right)^{2}},
$$

and thus we set

$$
a_{i j}=\frac{\left(k_{i}-k_{j}\right)^{2}}{\left(k_{i}+k_{j}\right)^{2}},
$$


that will be used in determination of 3-soliton solution. It is also noticed that resonant phenomena [3] does not occur as the phase shift term $a_{12}$ can never be 0 or $\infty$ for $\left|k_{1}\right| \neq\left|k_{2}\right|$.

For 3-soliton solution, the auxiliary function is taken as

$$
\begin{aligned}
f(x, t)= & 1+e^{\theta_{1}}+e^{\theta_{2}}+a_{12} e^{\theta_{1}+\theta_{2}}+a_{23} e^{\theta_{2}+\theta_{3}}+a_{13} e^{\theta_{1}+\theta_{3}}+a_{123} e^{\theta_{1}+\theta_{2}+\theta_{3}} \\
= & 1+e^{k_{1} x-a k_{1}^{3} t}+e^{k_{2} x-a k_{2}^{3} t}+e^{k_{3} x-a k_{3}^{3} t}+\frac{\left(k_{1}-k_{2}\right)^{2}}{\left(k_{1}+k_{2}\right)^{2}} e^{k_{1} x-a k_{1}^{3} t+k_{2} x-a k_{2}^{3} t}+ \\
& \frac{\left(k_{2}-k_{3}\right)^{2}}{\left(k_{2}+k_{3}\right)^{2}} e^{k_{2} x-a k_{2}^{3} t+k_{3} x-a k_{3}^{3} t}+\frac{\left(k_{1}-k_{3}\right)^{2}}{\left(k_{1}+k_{3}\right)^{2}} \times e^{k_{1} x-a k_{1}^{3} t+k_{3} x-a k_{3}^{3} t} \\
& +a_{123} e^{k_{1} x-a k_{1}^{3} t+k_{2} x-a k_{2}^{3} t+k_{3} x-a k_{3}^{3} t} .
\end{aligned}
$$

Continuing as before, we obtain the term

$$
a_{123}=a_{12} a_{23} a_{13}
$$

This proves that 3 -soliton solutions of (7) are derivable and can be obtained by substituting (21) into (13). In a series of papers [3, 4, 14, 22? ], it is confirmed that every solitonic system that has $N=3$ soliton solutions, it also has soliton solutions for $N \geq 4$, hence the system is integrable. This result shows that the system (7) is integrable and $N$-soliton solution for the system can be obtained for finite $N, N \geq 1$.

\section{Numerical Simulations}

This section presents the dynamics of solitonic system (7) in one spatial dimension for specific values of parameters, i.e., $a=1, b=1, c=1, k_{1}=1, k_{2}=2, k_{3}=3, R_{1}=1$. Figure 1 portrays 1-solition solution of (7) where 3D plots of $u$ and $v$ are shown in sub-figures $1 \mathrm{a}$ and $1 \mathrm{e}$ respectively. Profiles $1 \mathrm{~b}, 1 \mathrm{c}, 1 \mathrm{~d}$ demonstrate the progression of single soliton for component $u$ whereas for $v$, progression is displayed in $1 \mathrm{f}, 1 \mathrm{~g}$ and $1 \mathrm{~h}$ at time $t=0,1,2$.

In Figure 2, 3D plot of 2-soliton solutions $u$ and $v$ are shown in $2 \mathrm{a}$ and $2 \mathrm{e}$ whereas progression of $u$ and $v$ are demonstrated in $2 \mathrm{~b}, 2 \mathrm{c}, 2 \mathrm{~d}$ and $2 \mathrm{f}, 2 \mathrm{~g}$ and $2 \mathrm{~h}$ respectively at time $t=0,1,2$.

Sub-figures 3a and 3e presents 3D plot of 3-soliton solutions $u$ and $v$ respectively and advancement of solitons can be seen in $3 \mathrm{~b}, 3 \mathrm{c}, 3 \mathrm{~d}$ and $3 \mathrm{f}, 3 \mathrm{~g}$, $3 \mathrm{~h}$ for the same in figure 3 at time $t=0,0.66667,1.3333$.

\section{Conclusion}

A generalized coupled $\mathrm{KdV}$ model (1) is taken into consideration to test its integrability. Our results show that Painlevé property holds only for the condition $p=a, q=c, r=$ $b$. So a new P-type system (7) is obtained whose Painlevé integrabilty is tested by WTC method. To examine the complete integrability of the newly obtained system, simplified form of Hirota's bilinear test is performed. Consequently, three soliton solutions P-type KdV system are formally obtained and confirmed that $\mathrm{N}$-soliton solutions exist that lead to the complete integrability.

\section{Acknowledgement}

The first author would like to express sincere gratitude to the University Grants Commission for the financial assistance (Letter No. 19/06/2016(i)EU-V). 


\section{References}

[1] D.S. Wang, Applied Mathematics and Computation 216(4), 1349-1354 (2010).

[2] C.X. Li, Journal of the Physical Society of Japan 73(2), 327-331 (2004).

[3] R. Hirota, M. Ito, Journal of the Physical Society of Japan 52(3), 744-748 (1983).

[4] R. Hirota, Physical Review Letters 27(18), 1192 (1971).

[5] J. Hietarinta, Journal of Mathematical Physics 28(8), 1732-1742 (1987).

[6] A.A. Rady, E. Osman, M. Khalfallah, Applied mathematics and computation 210(1), 177-181 (2009).

[7] N.J. Zabusky, Chaos: An Interdisciplinary Journal of Nonlinear Science 15(1), 015102 (2005).

[8] S.F. Tian, Applied Mathematics Letters 100, 106056 (2020).

[9] A. Biswas, Physics Letters A 372(25), 4601-4602 (2008).

[10] A.S. Fokas, Physical Review Letters 96(19), 190-201 (2006).

[11] M.J. Ablowitz, M.A. Ablowitz, P.A. Clarkson, P.A. Clarkson, Solitons, nonlinear evolution equations and inverse scattering, 149 (Cambridge university press, 1991).

[12] M.J. Ablowitz, A. Ramani, H. Segur, Journal of Mathematical Physics 21(4), 715-721 (1980).

[13] M. Ablowitz, A. Ramani, H. Segur, Journal of Mathematical Physics 21(5), 1006-1015 (1980).

[14] W. Hereman, A. Nuseir, Mathematics and Computers in Simulation 43(1), 13-27 (1997).

[15] A.M. Wazwaz, Physica Scripta 83(1), 015002 (2010).

[16] A.M. Wazwaz, Central European Journal of Physics 9(3), 835-840 (2011).

[17] A.M. Wazwaz, Applied Mathematics and Computation 199(1), 133-138 (2008).

[18] A.M. Wazwaz, Physics Letters A 373(21), 1844-1846 (2009).

[19] J. Weiss, M. Tabor, G. Carnevale, Journal of Mathematical Physics 24(3), 522-526 (1983).

[20] X. Gui-Qiong, L. Zhi-Bin, Chinese Physics Letters 20(7), 975 (2003).

[21] S. Zhang, M. Chen, Mathematical Problems in Engineering 2015 (2015).

[22] J. Hietarinta, Journal of Mathematical Physics 28(9), 2094-2101 (1987). 